\title{
BREAKING AND MANAGING BAD NEWS TO CANCER PATIENT AND FAMILY: NURSING INTERVENTION
}

Mafalda Ferreira ${ }^{1}$; Patrícia Alves ${ }^{2}$

Lisbon Portuguese Oncology Institute, Francisco Gentil E.P.E., Medical Oncology \& Neurology ${ }^{1}$

Superior Nursing School of Lisbon, Nursing Fundamentals ${ }^{2}$

\section{Introduction}

The process of breaking and managing bad news in oncology is complex and susceptible of being a source of discomfort for all participants ${ }^{1}$. Cancer is still a pathology with negative connotations, related to warlike metaphors and death ${ }^{2}$. It is thus possible to exist a connotation of vulnerability associated to the care in oncology: of the patient and the family for all that they are experiencing and the need for constant readaptation ${ }^{3}$; of the health professionals, considering the training that the communication techniques demand, as well as for the need to manage their own emotions ${ }^{1}$. There are multiple inter-related factors that make the process of breaking and managing bad news a challenge ${ }^{4}$. Due to its proximity to the cancer patient and the family when providing care, nurses are a key element in this process, being essential to develop competencies in this area ${ }^{5}$.

\section{Objectives}

Develop skills in breaking and managing bad news to the cancer patient and family, as well as to promote the improvement of nursing care.

\section{Methods and Materials}

This analysis was based on a project methodology. Initially, the interest in this topic, associated with the scientific evidence consulted, contributed to the need of improvement of the practice in breaking and managing bad news to the cancer patient and family. Then, this process was also identified as a training need of the nurses of an Internal Medicine Ward (Charts 1 and 2). In this way, it was built a training and intervention project that involved carrying out an internship for three months in places considered having good practices in this subject: a Bone Marrow Transplantation Unit, a Palliative Care Unit and an Inpatient Interdisciplinary Palliative Consult Team. Throughout the course were applied questionnaires and constructed documents to support the practice. It was also made reflection on action using the Gibbs Reflective Cycle ${ }^{6}$, provided health care to cancer patient/family and performed training sessions to nurses. In these learning moments was used the S-P-I-K-E-S protocol, which is one of the most used protocols for breaking bad news ${ }^{5}$.

Chart 1: "Do you feel prepared for breaking bad news to the cancer patients and families to whom you provide health care?"

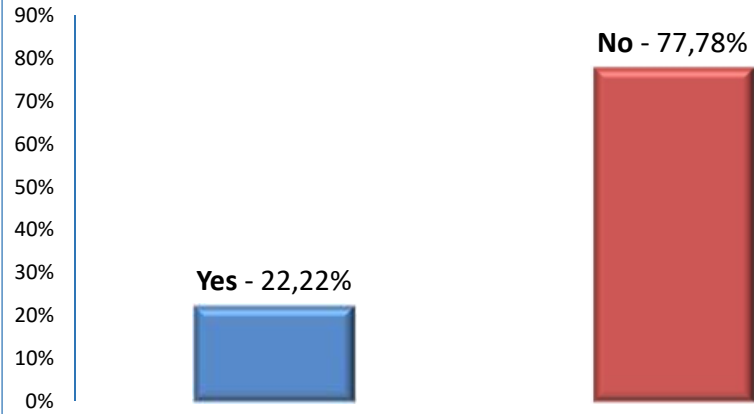

Chart 2: "Breaking bad news is susceptible to cause you: (possible to select more than one option)"

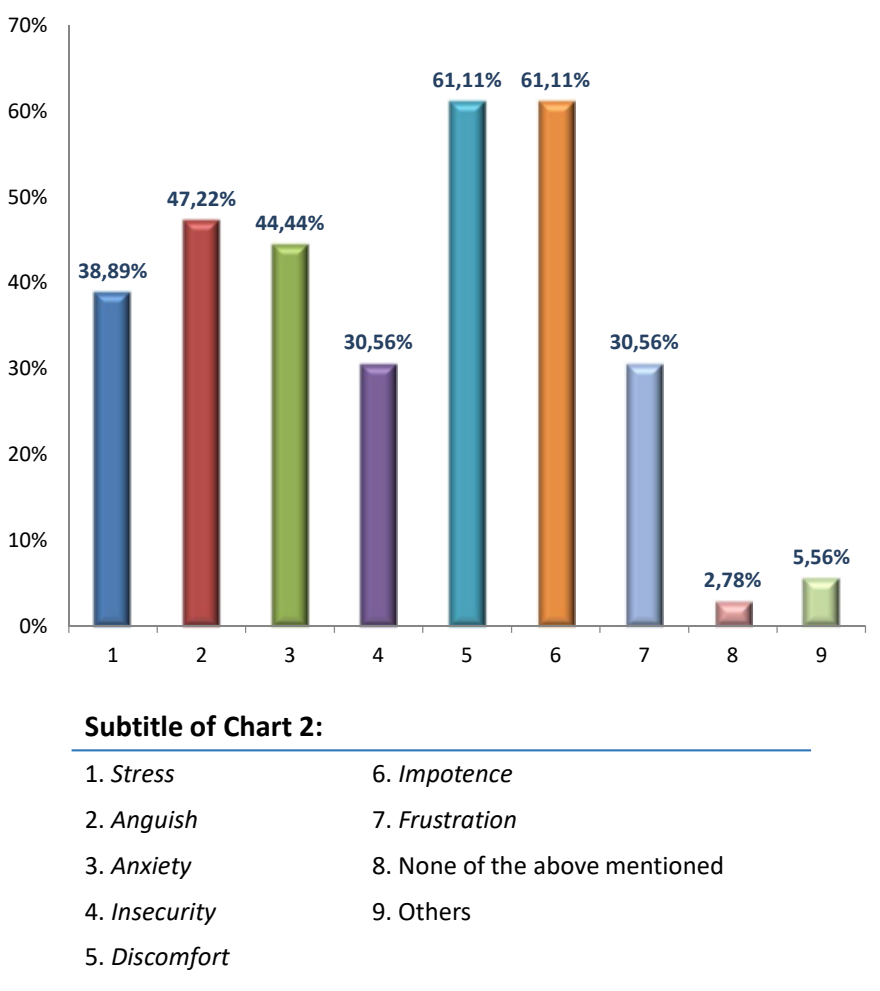

\section{Results}

Skills were acquired in the use of communication techniques, in the development of the relationship with cancer patient/family and in emotional management, allowing an advanced nursing practice. The nurses who received skills training considered the contributions positive to their practice: were enhanced the importance of knowledge about strategies and protocols of action in the scope of breaking and managing bad news, the clarification of the nurse's role in this process, the discussion of case studies and the reflection about the practice.

\section{Conclusion}

It is possible to develop skills in this area based on professional experience and using this methodology, as long as nurses are aware of their training needs and are available to reflect on action and to improve their practice. It is essential to have a larger investment in theoretical and practical training for the development of skills in breaking and managing bad news. The need for more scientific research in nursing dedicated to this subject is also vital. This research may focus on guiding strategies to be used, considering that S-P-I-K-E-S may be limiting in the scope of nursing intervention because it appears to be constructed for planned situations. Besides that, it could also be significant to develop research that evidences the relevance of coaching to health care outcomes and quality, as well as the importance of the articulation of the multidisciplinary team.

\section{Contact}

Mafalda Ferreira

Lisbon Portuguese Oncology Institute

Rua Prof. Lima Basto, 1099-023 Lisbon

mafalda.alexandra@gmail.com 00351912386213

\section{References}

C. Mukherjee, S. (2012). The Emperor of all Maladies: a biography of cancer. Lisboa: Bertrand Editora. Barbero, J., Barreto, P., Arranz, P., Bayés, R. (2005). Comunicación en oncologia clinica. Madrid: Editorial Just in Time S. Warnock, C., Buchanan, I. \& Tod, A. (2017). The difficulties experienced by nurses and healthcare staff involved in the process of breaking bad news. Journal of Advanced Nursing, 73 (7), p. $1632-1645.4$
Warnock, c. (2014). Breaking bad news: issues relating to nursing practice. Nursing Standard, 28 (45), p. $51-58$ 